PROCEEDINGS OF THE

AMERICAN MATHEMATICAL SOCIETY

Volume 140, Number 2, February 2012, Pages 635-644

S 0002-9939(2011)10930-4

Article electronically published on June 17, 2011

\title{
THE KDV CURVE AND SCHRÖDINGER-AIRY CURVE
}

\author{
CHONG SONG
}

(Communicated by Jianguo Cao)

\begin{abstract}
Among other things, we introduce the notion of $\mathrm{KdV}$ curves and Schrödinger-Airy curves. These curves are stable solutions to the geometric KdV-Airy flow equation and Schrödinger-Airy flow equation respectively, which were recently proposed by Sun and Wang. We demonstrate that the $\mathrm{KdV}$ curves can be regarded as a 3rd-order analogue of geodesics. Other interesting properties of these curves will be addressed. Explicit examples of these curves will be provided. In addition, we will consider a perturbed $\mathrm{KdV}$ curve system and show the existence of multiple solutions to this system on the torus.
\end{abstract}

\section{The KdV CuRve}

Suppose that $(N, \omega, J)$ is a Kähler manifold with symplectic form $\omega$ and complex structure $J$ and that $u(x, t)$ is a smooth map from $S^{1} \times \mathbb{R}^{1}$ into $N$. Let $\nabla_{x}$ denote the covariant derivative $\nabla_{\frac{\partial}{\partial x}}$ on the pull-back bundle $u^{-1} T N$ induced from the Levi-Civita connection $\nabla$ on $N$. In [5], Sun and Wang introduced the so-called geometric KdV-Airy flow as follows:

$$
u_{t}=\nabla_{x}^{2} u_{x}+\frac{1}{2} R\left(u_{x}, J u_{x}\right) J u_{x}
$$

where $R$ is the curvature tensor on $N$. Here we denote $u_{t}=\nabla_{t} u, u_{x}=\nabla_{x} u$ and write $J=J(u)$ for simplicity. Equation (1.1) is a geometric flow which stems from the vortex filament dynamics and belongs to the same family as the Schrödinger flow (cf. 44):

$$
u_{t}=J \nabla_{x} u_{x}
$$

In certain circumstances, equation (1.1) transforms into the well-known modified $\mathrm{KdV}$ equation. We refer to [5] for more details and background knowledge.

The $K d V$ curve is defined as the stable solution to equation (1.1). Namely, a map $u \in C^{\infty}\left(S^{1}, N\right)$ is called a $\mathrm{KdV}$ curve if it satisfies

$$
\nabla_{x}^{2} u_{x}+\frac{1}{2} R\left(u_{x}, J u_{x}\right) J u_{x}=0 .
$$

Suppose $N$ is embedded in a Euclidean space $\mathbb{R}^{K}$. Define the KdV energy functional on Sobolev space,

$$
W=W^{2,2}\left(S^{1}, N\right):=\left\{W^{2,2}\left(S^{1}, \mathbb{R}^{K}\right) \mid u(x) \in N \text { for a.e. } x \in S^{1}\right\},
$$

Received by the editors October 22, 2010 and, in revised form, November 28, 2010 and December 2, 2010.

2010 Mathematics Subject Classification. Primary 37K25, 58E50, 53C99.

(c) 2011 American Mathematical Society Reverts to public domain 28 years from publication 
as follows:

$$
F(u)=\frac{1}{2} \int_{S^{1}}\left\langle\nabla_{x} u_{x}, J u_{x}\right\rangle d x .
$$

Let $u_{s}:[0, \delta] \rightarrow W$ be a variation of $u$ and

$$
\left.\frac{\partial u_{s}}{\partial s}\right|_{s=0}=\xi \in T_{u} W=W^{2,2}\left(S^{1}, T_{u} N\right) .
$$

Then a direct computation yields

$$
(d F(u), \xi)=\left.\frac{d}{d s} F\left(u_{s}\right)\right|_{s=0}=\int_{S^{1}}\left\langle J \nabla_{x}^{2} u_{x}+\frac{1}{2} R\left(J u_{x}, u_{x}\right) u_{x}, \xi\right\rangle .
$$

So the KdV curves are actually critical points of the functional $F$.

There is a symplectic form $\Omega$ on $W$ naturally induced by the symplectic form $\omega$ on $N$. Indeed, for any vector fields $X, Y \in T_{u} W$, we can define

$$
\Omega(X, Y)=\int_{S^{1}} \omega(X(u), Y(u)) d x
$$

Similarly, there is an induced complex structure on $W$, which we still denote by $J$. The Hamiltonian vector field $X_{F}$ associated with $F$ is defined by

$$
\Omega\left(X_{F}, \cdot\right)=d F .
$$

Then the KdV-Airy flow (1.1) can be written in the following form:

$$
u_{t}=X_{F}=J \nabla F(u),
$$

which is the Hamiltonian flow of $F$ on the infinite dimensional symplectic manifold $(W, \Omega)$. On the other hand, the Schrödinger flow (1.2) is known to be the Hamiltonian flow of the normal energy functional

$$
E(u)=\frac{1}{2} \int_{S^{1}}\left|u_{x}\right|^{2} d x,
$$

and the stable solutions for Schrödinger flow are just geodesics. It turns out that the KdV-Airy flow and the Schrödinger flow belong to the same integrable system [5]. Therefore, the KdV curves are not only important for understanding the KdV-Airy flow, but are also of special interest as a higher order analogue of geodesics.

It is a basic fact that geodesics have constant speed. In other words, the energy density $e(u)=\left|u_{x}\right|^{2}$ remains the same along geodesics. There is an analogous result for $\mathrm{KdV}$ curves.

Theorem 1.1. For any given $K d V$ curve $u$, the quantity

$$
f(u)=\left\langle\nabla_{x} u_{x}, J u_{x}\right\rangle
$$

is a constant.

Proof. A simple calculation yields

$$
\begin{aligned}
d_{x} f(u) & =d_{x}\left\langle\nabla_{x} u_{x}, J u_{x}\right\rangle \\
& =\left\langle\nabla_{x}^{2} u_{x}, J u_{x}\right\rangle+\left\langle\nabla_{x} u_{x}, J \nabla_{x} u_{x}\right\rangle \\
& =-\frac{1}{2}\left\langle R\left(u_{x}, J u_{x}\right) J u_{x}, J u_{x}\right\rangle \\
& =0 .
\end{aligned}
$$

In fact, on some manifolds, KdV curves are just geodesics. 
Theorem 1.2. If the holomorphic sectional curvature $k$ of $N$ is non-positive, the $K d V$ curves are geodesics. In particular, if $k$ is strictly negative, then the $K d V$ curves are just constant maps.

Proof. On manifolds with non-positive holomorphic sectional curvature, we have

$$
k(X)=\frac{R(X, J X, J X, X)}{|X|^{4}} \leq-\delta, \quad \delta \geq 0 .
$$

For any KdV curve $u$ which satisfies equation (1.3), multiplying by $u_{x}$ and integrating by parts, we have

$$
\begin{aligned}
0 & =\int\left\langle\nabla_{x}^{2} u_{x}, u_{x}\right\rangle+\frac{1}{2} \int R\left(u_{x}, J u_{x}, J u_{x}, u_{x}\right) \\
& =-\int\left|\nabla_{x} u_{x}\right|^{2}+\frac{1}{2} \int k\left(u_{x}\right)\left|u_{x}\right|^{4} \\
& \leq-\int\left|\nabla_{x} u_{x}\right|^{2}-\frac{1}{2} \delta \int\left|u_{x}\right|^{4} .
\end{aligned}
$$

This implies that $\nabla_{x} u_{x}=0$, which means that $u$ is a geodesic. Moreover, if $\delta>0$, then $u_{x} \equiv 0$, which means that $u$ is a constant map.

\section{SCHRÖDINGER-AIRY CURVE}

In 5], the authors also addressed the so-called Schrödinger-Airy flow, which can be regarded as a geometric generalization of the Hirota equation. Explicitly, the flow is defined as follows:

$$
u_{t}=\alpha J \nabla_{x} u_{x}+\beta\left(\nabla_{x}^{2} u_{x}+\frac{1}{2} R\left(u_{x}, J u_{x}\right) J u_{x}\right),
$$

where $\alpha$ and $\beta$ are two positive numbers. We call a stable solution to equation (2.1) a Schrödinger-Airy curve. Namely, a Schrödinger-Airy curve is a map $u \in C^{\infty}\left(S^{1}, N\right)$ which satisfies the equation

$$
\nabla_{x}^{2} u_{x}+\frac{1}{2} R\left(u_{x}, J u_{x}\right) J u_{x}=\lambda J \nabla_{x} u_{x}
$$

where $\lambda$ is a function on $S^{1}$. It's a natural extension of the KdV curve. Particularly, a KdV curve satisfies equation (2.2) for $\lambda \equiv 0$. If we regard $\lambda$ as a Lagrange multiplier, then the Schrödinger-Airy curve is a critical point of $F(u)$ under the constraint $E(u)=$ const.

Note that both the KdV curve and the Schrödinger-Airy curve can be defined weakly in $W^{2,2}\left(S^{1}, N\right)$. In fact, we may assume that the compact manifold $N$ is embedded in a Euclidean space $\mathbb{R}^{K}$. Denote the inner product on $\mathbb{R}^{K}$ by $(\cdot, \cdot)$ and the second fundamental form of $N$ by $A$. Then we have

$$
\nabla_{x} u_{x}=u_{x x}-A(u)\left(u_{x}, u_{x}\right) .
$$

It follows that

$$
\begin{aligned}
\nabla_{x}^{2} u_{x} & =\left(\nabla_{x} u_{x}\right)_{x}-A(u)\left(u_{x}, \nabla_{x} u_{x}\right) \\
& =u_{x x x}-\left[A(u)\left(u_{x}, u_{x}\right)\right]_{x}-A(u)\left(u_{x}, \nabla_{x} u_{x}\right) \\
& =u_{x x x}-\nabla A(u)\left(u_{x}, u_{x}, u_{x}\right)-3 A(u)\left(u_{x}, \nabla_{x} u_{x}\right) .
\end{aligned}
$$


Therefore, we may call a map $u \in W^{2,2}\left(S^{1}, N\right)$ a weak $\mathrm{KdV}$ curve if $u$ is a $W^{2,2}$ weak solution of equation (1.3). Namely, $u$ is a weak KdV curve if for any $v \in$ $C^{\infty}\left(S^{1}, \mathbb{R}^{K}\right)$, it follows that

$$
\int_{S^{1}}\left(\nabla_{x} u_{x}, v_{x}\right) d x+\int_{S^{1}}\left(A(u)\left(u_{x}, \nabla_{x} u_{x}\right), v\right) d x-\frac{1}{2} \int_{S^{1}}\left(R\left(u_{x}, J u_{x}\right) J u_{x}, v\right)=0 .
$$

Similarly a weak Schrödinger-Airy curve is defined as a $W^{2,2}$-weak solution to equation (2.2). The following theorem shows that a weak Schrödinger-Airy (or $\mathrm{KdV}$ ) curve is actually smooth.

Theorem 2.1. A $W^{2,2}$-weak Schrödinger-Airy curve is smooth.

Proof. By (2.3), equation (2.2) can be rewritten as

$$
u_{x x x}=f\left(u_{x}, u_{x x}\right)
$$

where

$$
\begin{aligned}
f\left(u_{x}, u_{x x}\right)= & \nabla A(u)\left(u_{x}, u_{x}, u_{x}\right)+3 A(u)\left(u_{x}, \nabla_{x} u_{x}\right) \\
& -\frac{1}{2} R\left(u_{x}, J u_{x}\right) J u_{x}+\lambda J \nabla_{x} u_{x} .
\end{aligned}
$$

It is obvious that equation (2.4) is an elliptic equation for $u_{x}$, and $u_{x}$ is a $W^{1,2}$ weak solution to (2.4) if $u$ is a $W^{2,2}$-weak solution to (2.2). By Sobolev embedding theorems, $u \in W^{2,2}\left(S^{1}, N\right)$ implies $u_{x} \in C^{\alpha}$ for some $\alpha \in(0,1)$. Hence $f\left(u_{x}, u_{x x}\right) \in$ $L^{2}$. Now by the standard $L^{2}$-estimate, it follows that $u_{x} \in W^{2,2}$ from equation (2.4). A bootstrapping argument then proves the theorem.

In the remaining part of this section, we always suppose that $N$ is a closed Riemann surface. In this case, we shall show that Schrödinger-Airy curves satisfy some nice properties. In particular, all these properties hold for KdV curves.

First we observe that the functional $F$ is not a geometric invariant. That is, it depends on the choice of the parameter of the curve $u$. Let $s=\int_{0}^{x}\left|u_{x}\right| d x$ be the arc length parameter of $u$. Let $\mathbf{t}=u_{s}$ be the unit tangent vector and $\mathbf{n}$ be the unit normal vector orthogonal to $\mathbf{t}$. We denote $\nabla_{s}=\nabla_{u_{s}}$. Then

$$
\nabla_{s} u_{s}=k_{g} \mathbf{n}
$$

where $k_{g}$ is the geodesic curvature. It follows that

$$
\left\langle\nabla_{s} u_{s}, J u_{s}\right\rangle=\left\langle k_{g} \mathbf{n}, J \mathbf{t}\right\rangle=k_{g}
$$

Note that $k_{g}$ in the last equality may vary from the usual definition of geodesic curvature by a minus sign, depending on the complex structure $J$. But here we only care about the absolute value of $k_{g}$ and ignore the slight difference of sign. On the other hand, $d s=\left|u_{x}\right| d x$. Therefore, we get

$$
\left\langle\nabla_{x} u_{x}, J u_{x}\right\rangle=\left|u_{x}\right|^{3}\left\langle\nabla_{s} u_{s}, J u_{s}\right\rangle+\left|u_{x}\right|^{2} \nabla_{s}\left|u_{x}\right|\left\langle u_{s}, J u_{s}\right\rangle=\left|u_{x}\right|^{3} k_{g} .
$$

Theorem 2.2. Suppose $N$ is a Riemann surface and u is a Schrödinger-Airy curve. If $u$ has constant speed $\left|u_{x}\right|=c$, then $u$ has constant geodesic curvature.

Proof. Multiplying equation (2.2) by $J u_{x}$, we get

$$
\left\langle\nabla_{x}^{2} u_{x}, J u_{x}\right\rangle=-\lambda\left\langle\nabla_{x} u_{x}, u_{x}\right\rangle .
$$

By (2.5), the geodesic curvature $k_{g}$ satisfies

$$
\left\langle\nabla_{x} u_{x}, J u_{x}\right\rangle=\left|u_{x}\right|^{3} k_{g} .
$$


Note that

$$
\nabla_{x}\left\langle\nabla_{x} u_{x}, J u_{x}\right\rangle=\left\langle\nabla_{x}^{2} u_{x}, J u_{x}\right\rangle .
$$

Hence equality (2.6) is equivalent to

$$
\nabla_{x}\left(\left|u_{x}\right|^{3} k_{g}\right)=-\frac{\lambda}{2} \nabla_{x}\left|u_{x}\right|^{2} .
$$

It is easy to see that if $\left|u_{x}\right|=c$ is a constant, then $\nabla_{x} k_{g}=0$, as desired.

Next we suppose in addition that $N$ has constant curvature. We show that in this case the geodesic curvature can be computed.

Theorem 2.3. Suppose $N$ is a Riemann surface with constant curvature $k$ and $u$ is a non-trivial Schrödinger-Airy curve. Assume $u$ has constant speed $\left|u_{x}\right|=c$. Then the geodesic curvature $k_{g}$ is determined by $\lambda$.

Proof. For a constant curvature surface $N$, we have

$$
R\left(u_{x}, J u_{x}\right) J u_{x}=k\left|u_{x}\right|^{2} u_{x} .
$$

Since $u$ has constant speed, we have

$$
0=\nabla_{x}\left|u_{x}\right|^{2}=\left\langle\nabla_{x} u_{x}, u_{x}\right\rangle
$$

Thus we may suppose $\nabla_{x} u_{x}=\alpha J u_{x}$ for some function $\alpha(x)$. It follows from (2.5) that

$$
\left\langle\nabla_{x} u_{x}, J u_{x}\right\rangle=-\left|u_{x}\right|^{2} \alpha=\left|u_{x}\right|^{3} k_{g} .
$$

Hence $\alpha=-c k_{g}$, which is constant by Theorem 2.2. Consequently, we have

$$
\nabla_{x} u_{x}=-c k_{g} J u_{x}, \quad \nabla_{x}^{2} u_{x}=-c^{2} k_{g}^{2} u_{x} .
$$

Then using equations (2.2) and (2.7), we get

$$
\begin{aligned}
\nabla_{x}^{2} u_{x}+\frac{1}{2} R\left(u_{x}, J u_{x}\right) J u_{x} & =-c^{2} k_{g}^{2} u_{x}+\frac{1}{2} c^{2} k u_{x} \\
& =\lambda J \nabla_{x} u_{x}=\lambda c k_{g} u_{x} .
\end{aligned}
$$

This simplifies to

$$
k_{g}^{2}+\frac{\lambda}{c} k_{g}-\frac{1}{2} k=0 .
$$

This is a second order equation for $k_{g}$, and the solution is given by

$$
k_{g}=\frac{\lambda \pm c \sqrt{\lambda^{2}+2 k c^{2}}}{2 c},
$$

provided $\lambda^{2}+2 k c^{2} \geq 0$.

\section{EXAMPLES}

In this section let's see some examples of Schrödinger-Airy curves on Riemann surfaces.

Example 3.1. On the Euclidean plane, the round circles are Schrödinger-Airy curves for appropriate $\lambda$. 
Example 3.2. On the standard sphere $S^{2}$, except the great circle, any non-trivial intersection of a plane in $\mathbb{R}^{3}$ and the sphere $S^{2}$ gives rise to a Schrödinger-Airy curve.

For example, let $u: S^{1} \rightarrow S^{2}$ be given by

$$
u(x)=(\cos x \cos \phi, \sin x \cos \phi, \sin \phi), \quad x \in[0,2 \pi) .
$$

Here we suppose $\phi \in(0, \pi / 2) \cup(\pi / 2, \pi)$. That is, we exclude trivial maps and the geodesics. Then a direct computation yields:

$$
\begin{aligned}
u_{x}(x) & =(-\sin x, \cos x, 0) \cos \phi, \\
\nabla_{x} u_{x}(x) & =(-\cos x \sin \phi,-\sin x \sin \phi, \cos \phi) \sin \phi \cos \phi, \\
\nabla_{x}^{2} u_{x}(x) & =-\left(\sin ^{2} \phi\right) u_{x} .
\end{aligned}
$$

Since the sphere has constant curvature 1 , it follows that

$$
R\left(u_{x}, J u_{x}\right) J u_{x}=\left|u_{x}\right|^{2} u_{x}=\left(\cos ^{2} \phi\right) u_{x} .
$$

On the other hand, the complex structure on $S^{2}$ is given by $J(u)=u \times$. Thus

$$
J(u) \nabla_{x} u_{x}=-(\sin x,-\cos x, 0) \sin \phi \cos \phi=-(\sin \phi) u_{x} .
$$

Therefore, $u$ is a Schrödinger-Airy curve with

$$
\lambda=\frac{\sin ^{2} \phi-\frac{1}{2} \cos ^{2} \phi}{\sin \phi} .
$$

Particularly, if we choose $\phi=\arccos \sqrt{\frac{2}{3}}$, we get a non-trivial KdV curve.

Let us now turn our attention to the hyperbolic plane.

Example 3.3. Let $g_{-1}=d r^{2}+(\sinh r)^{2} d \theta^{2}$ be the hyperbolic metric of constant curvature -1 on $\mathbb{R}^{2}$. We consider the curve $u: S^{1} \rightarrow \mathbb{R}^{2}$ given by $u(x)=(r, x)$. It is clear that $\left|u_{x}\right|=\sinh r$ and

$$
\nabla_{x} u_{x}=-(\sinh r)(\cosh r) \frac{\partial}{\partial r}=(\cosh r) J u_{x} .
$$

Using this equation together with (2.2), we observe that

$$
\begin{aligned}
-\lambda(\cosh r) u_{x} & =\lambda J \nabla_{x} u_{x}=\nabla_{u_{x}}^{2} u_{x}+\frac{1}{2} R\left(u_{x}, J u_{x}\right) J u_{x} \\
& =\nabla_{x}\left[(\cosh r) J u_{x}\right]-\frac{1}{2}\left|u_{x}\right|^{2} u_{x} \\
& =\left[-(\cosh r)^{2}-\frac{1}{2}(\sinh r)^{2}\right] u_{x} .
\end{aligned}
$$

It follows that

$$
\lambda=\frac{(\cosh r)^{2}+\frac{1}{2}(\sinh r)^{2}}{\cosh r} .
$$

Note that $u$ has constant geodesic curvature $\cosh r / \sinh r$. Consequently, there are no non-trivial KdV curves on a hyperbolic plane. 


\section{Perturbed KdV curves on $T^{2 n}$}

By Theorem 1.2, the KdV curves on non-positively curved manifolds are trivial, so it is natural to consider the perturbed system. Namely, we shall consider the following system:

$$
\nabla_{x}^{2} u_{x}+\frac{1}{2} R\left(u_{x}, J u_{x}\right) J u_{x}=-J(u) \nabla_{u} H(x, u),
$$

where $H(x, u)$ is a Hamiltonian function defined on $S^{1} \times N$. Obviously, solutions to the above system are critical points of the perturbed functional

$$
F_{H}(u)=F(u)+\int_{S^{1}} H(x, u) d x .
$$

This is a strongly indefinite functional which has no lower bound. In general it is very hard to find critical points of $F_{H}$. Fortunately, in the special case of the torus, this problem can be significantly simplified.

In the following context, we suppose that $N=T^{2 n}$ is the $2 n$-dimensional flat torus and use $t$ to denote the variable in $S^{1}$ instead of $x$. Then the system (4.1) is reduced to

$$
\frac{d^{3} u}{d t^{3}}=-J \nabla H_{t}(u), \quad t \in S^{1} .
$$

System (4.2) is similar to the usual Hamiltonian system

$$
\frac{d u}{d t}=-J \nabla H_{t}(u)
$$

It is well known that the solution to (4.3) corresponds to the fixed point of symplectomorphisms on $T^{2 n}$, and the number of solutions is related to the famous Arnold conjecture, which is solved by Conley and Zehnder [3]. The method they used relies on a saddle point reduction, which is due to Amann [1. Namely, finding periodic solutions to the infinite dimensional system (4.3) shifts to a finite dimensional variational problem by a Lyapunov-Schmidt reduction, and the Morse theory can be applied.

The same saddle point reduction can be applied to our system (4.2). But here we follow a simplified version which appears in Chang's book [2]. Our main result is the following:

Theorem 4.1. Suppose $H_{t}(u)=H(t, u) \in C^{2}\left(S^{1} \times T^{2 n}, \mathbb{R}^{1}\right)$. Then the system (4.2) has at least $2 n+1$ solutions. If in addition all solutions are non-degenerate, then there are $2^{2 n}$ solutions to (4.2).

Before proving the theorem, let us first observe some basic facts. The operator

$$
A:=J \frac{d^{3}}{d t^{3}}
$$

is defined on a dense subspace $D(A)$ of the Hilbert space

$$
L:=L^{2}\left(S^{1}, T^{2 n}\right) .
$$

Any function in $L$ can be viewed as a periodic function in $L^{2}\left(S^{1}, \mathbb{R}^{2 n}\right)$. By Fourier series theory, a function $u \in L^{2}\left(S^{1}, \mathbb{R}^{2 n}\right)$ can always be decomposed into

$$
u(t)=\sum_{j=1}^{n} \sum_{m=-\infty}^{+\infty} c_{m j} e^{-i m t} \phi_{j},
$$


where $\left\{\phi_{1}, \cdots, \phi_{n}\right\}$ is a basis of $\mathbb{C}^{n}=\mathbb{R}^{2 n}$ and $c_{m j}$ satisfies

$$
\sum_{m=-\infty}^{+\infty}\left|c_{m j}\right|^{2}<\infty, j=1, \cdots, 2 n
$$

Since

$$
J \frac{d^{3}}{d t^{3}}\left(e^{-i m t} \phi_{j}\right)=-m^{3} e^{-i m t} \phi_{j},
$$

it is obvious that $A$ is a selfadjoint operator which only has point spectrum $\left\{-m^{3} ; m \in \mathbb{Z}\right\}$, and the eigenspace of the eigenvalue $\lambda=-m^{3}$ is

$$
\Lambda\left(-m^{3}\right)=\operatorname{span}\left\{e^{-i m t} \phi_{1}, \cdots, e^{-i m t} \phi_{n}\right\} .
$$

We shall work on the space $V=D\left(|A|^{\frac{1}{2}}\right)$ defined by

$$
V:=\left\{v \in L: \sum_{j=1}^{n} \sum_{m=-\infty}^{+\infty}\left(1+|m|^{3}\right)\left|c_{m j}\right|^{2}<\infty\right\} .
$$

There is a well-defined functional on $V$ given by

$$
a(v)=\frac{1}{2} \int_{S^{1}}\langle A v, v\rangle d t-\int_{S^{1}} H_{t}(v) d t, v \in V .
$$

Just as in the theory of the Hamiltonian system (4.3), it easy to verify that the Euler-Lagrange equation of $a$ is exactly the system (4.2). A solution $v$ is called non-degenerate if the Hessian $d^{2} a(v)$ is an isomorphism.

Now we are ready to prove Theorem 4.1 .

Proof of Theorem 4.1. Since the solutions to equation (4.2) are just critical points of the functional $a \in C^{2}\left(V, \mathbb{R}^{1}\right)$, we only have to investigate the number of critical points of $a$. We prove this theorem in two steps. First, we show that the required critical points of $a$ are in one-one correspondence with a function $h$, which is defined on a finite dimensional space. Then we show that $h$ satisfies the P.S. condition, so that the standard Morse theory yields the desired conclusion.

As previously noted, the selfadjoint operator $A$ only has a discrete spectrum. So there exists $\epsilon>0$ small, such that $-\epsilon$ is not in the spectrum and $A_{\epsilon}:=\epsilon I+A$ is invertible.

On the other hand, since the Hamiltonian $H(t, u) \in C^{2}\left(S^{1} \times T^{2 n}\right)$ is defined on a compact space, we may suppose that there exists a real number $B>0$ such that

$$
\left\|\nabla_{u}^{2} H_{t}\right\| \leq B, \forall t \in S^{1} .
$$

The following step is to decompose the domain space $V=D\left(|A|^{\frac{1}{2}}\right)$. First, for each eigenvalue $\lambda$ of $A$, let $P(\lambda)$ be the projection from $L$ to the eigenspace $\Lambda(\lambda)$. Let

$$
P_{0}=\sum_{-B \leq \lambda \leq B} P(\lambda), P_{+}=\sum_{\lambda<-B} P(\lambda), P_{-}=\sum_{\lambda>B} P(\lambda),
$$

and let $L_{0}=P_{0} L, L_{ \pm}=P_{ \pm} L$. We also denote

$$
E_{+}=\sum_{\lambda>0} P(\lambda), E_{-}=\sum_{\lambda<0} P(\lambda) .
$$

Next, using the invertible operator $A_{\epsilon}$, we may decompose the space $V=D\left(|A|^{\frac{1}{2}}\right)$ into

$$
V=V_{0} \oplus V_{+} \oplus V_{-},
$$


where $V_{0}=\left|A_{\epsilon}\right|^{-\frac{1}{2}} L_{0}, V_{ \pm}=\left|A_{\epsilon}\right|^{-\frac{1}{2}} L_{ \pm}$. Define the graph norm on $V$ by

$$
\|v\|_{V}^{2}:=\left\||A|^{\frac{1}{2}} v\right\|_{L}^{2}+\epsilon^{2}\|v\|_{L}^{2} .
$$

Then for any $u \in L$ and $v=\left|A_{\epsilon}\right|^{-\frac{1}{2}} u \in V$, we have $\|u\|_{L}=\|v\|_{V}$. Moreover, we have the following decomposition:

$$
u=u_{+}+u_{-}+u_{0}, \quad v=v_{+}+v_{-}+v_{0},
$$

where $u_{0}=P_{0} u, u_{ \pm}=P_{ \pm} u$, and $v_{0}=\left|A_{\epsilon}\right|^{-\frac{1}{2}} u_{0}, v_{ \pm}=\left|A_{\epsilon}\right|^{-\frac{1}{2}} u_{ \pm}$.

Now we define a functional on $L$ as follows:

$$
b(u)=\frac{1}{2}\left(\left\|u_{+}\right\|_{L}^{2}+\left\|E_{+} u_{0}\right\|_{L}^{2}-\left\|E_{-} u_{0}\right\|_{L}^{2}-\left\|u_{-}\right\|_{L}^{2}\right)-\Phi_{\epsilon}(v),
$$

where

$$
\Phi_{\epsilon}(v)=\frac{\epsilon}{2}\|v\|_{L}^{2}+\int_{S^{1}} H_{t}(v) d t
$$

Obviously, this functional $b$ is actually the same as the functional $a$ given by (4.5) such that $a(v)=b(u)$. Besides, $u$ is a critical point of $b$ if and only if $v$ is a critical point of $a$. The Euler-Lagrange equation of $b$ for $u_{ \pm}$is

$$
u_{ \pm}= \pm\left|A_{\epsilon}\right|^{-\frac{1}{2}} P_{ \pm} F_{\epsilon}(v),
$$

where

$$
F_{\epsilon}=\epsilon I+\nabla H_{t} \in C^{1}(V, V) .
$$

However, equation (4.6) is equivalent to

$$
v_{ \pm}=A_{\epsilon}^{-1} P_{ \pm} F_{\epsilon}\left(v_{+}+v_{-}+v_{0}\right) .
$$

A direct computation shows that the operator

$$
\mathcal{F}_{ \pm}:=A_{\epsilon}^{-1} P_{ \pm} F_{\epsilon} \in C^{1}(V, V)
$$

is a contraction. It follows by the implicit function theorem that there exists a solution $v_{ \pm}\left(v_{0}\right)$ to equation (4.7) for fixed $v_{0} \in V_{0}$. Thus we may define a functional on $V_{0}$ by

$$
h(z)=a(v(z))=a\left(v_{+}(z)+v_{-}(z)+z\right), z \in V_{0} .
$$

One verifies readily that $z$ is a critical point of $h$ if and only if $v(z)$ is a critical point of $a$. As a consequence, the problem of finding critical points of $a$ is now reduced to finding critical points of a $C^{2}$ functional $h$ defined on a finite dimensional space $V_{0}$.

To proceed, we decompose $V_{0}$ into $V_{0}=Z_{\star} \oplus Z_{0}$, where $Z_{\star}=E_{+} V_{0} \oplus E_{-} V_{0}$, and $Z_{0}$ is the kernel of $A$. Let $z=z_{\star}+z_{0}$, where $z_{\star} \in Z_{\star}, z_{0} \in Z_{0}$. Because $H_{t}$ is periodic in $v$ and $v_{ \pm}$is periodic in $z$ by equation (4.7), it follows that $h(z)$ is periodic in $z_{0}$. Note that $Z_{0}=\Lambda(0)$ is a $2 n$-dimensional space by (4.4). So $h(z)$ may be viewed as a functional defined on $Z_{\star} \times T^{2 n}$. If we let

$$
g(z)=\frac{1}{2}\left(A\left(v_{+}(z)+v_{-}(z)\right), v_{+}(z)+v_{-}(z)\right)-\int_{S^{1}} H_{t}(v(z)) d t,
$$

then the functional $h$ is in the following form:

$$
h(z)=h\left(z_{\star}, z_{0}\right)=\frac{1}{2}\left(A z_{\star}, z_{\star}\right)+g\left(z_{\star}, z_{0}\right) .
$$

It is easy to see that $d g(z)=P_{0} \nabla H_{t}(v(z))$ is bounded. Now by a standard argument in Morse theory (for example, see Theorem 5.3 in [2]), $f$ satisfies the P.S. condition 
and Theorem 4.1 is proved, provided the cuplength of $T^{2 n}$ is $2 n$ and the sum of the Betti numbers is $2^{2 n}$.

\section{ACKNOWLEDGEMENTS}

The author would like to express his deep gratitude to Prof. Youde Wang for giving him this interesting problem and for his continuous support. He would also like to thank Prof. Jianguo Cao and Prof. Meiyue Jiang for inspiring discussions and helpful advice.

\section{REFERENCES}

[1] H. Amann; Saddle points and multiple solutions of differential equations, Math. Z. 169 (1979), 122-166. MR 550724 (80j:47078)

[2] K.C. Chang; Infinite-dimensional Morse theory and multiple solution problems, Progress in Nonlinear Differential Equations 6, Birkhäuser (1993). MR:1196690(94e:58023)

[3] C. Conley and E. Zehnder; The Birkhoff-Lewis fixed point theorem and a conjecture of V. I. Arnold, Invent. Math. 73 (1983), 33-49. MR707347 (85e:58044)

[4] W. Y. Ding, Y. D. Wang; Schrödinger flows of maps into symplectic manifolds, Sci. China A41 (7), 746-755 (1998). MR.1633799 (99m:58062)

[5] X. W. Sun and Y. D. Wang; KdV geometric flows on Kähler manifolds, International Journal of Mathematics, to appear.

LMam, School of Mathematical Sciences, Peking University, Beijing 100871, PeoPLE'S REPUBLIC OF CHINA

E-mail address: songchong@amss.ac.cn 